\title{
A KOROVKIN TYPE APPROXIMATION THEOREM FOR BALÁZS TYPE BLEIMANN, BUTZER AND HAHN OPERATORS VIA POWER SERIES STATISTICAL CONVERGENCE
}

\author{
DİLEK SÖYLEMEZ \\ (Communicated by Tomasz Natkaniec)
}

\begin{abstract}
In this paper, we obtain a Korovkin type approximation theorem for power series statistical convergence of functions belonging to the class produced by multivariable modulus of continuity function. As an application of this theorem, we construct a non-tensor product Balázs type BBH operator which does not converge in ordinary sense. Moreover, we study promised approximation properties of this operator and compute the rate of convergence. Finally, we prove that our new approximation result works but its classical case fails.

\section{Preliminaries}

Summability theory has many applications in probability limit theorems, approximation theory and differential equations etc. ([8, 17, 19,30$])$. In Korovkin type approximation theory [2, by using summability methods may be beneficial when a sequence of positive linear operators does not converge to the identity operator in ordinary sense. In this direction, the first Korovkin type theorem was given by considering the concept of statistical convergence by Gadjiev and Orhan in [17. Following that study many authors gave several approximation results via summability theory (see, e.g., 3, 31, 12, 21, 27, 28, 31]).

Let $x=\left(x_{j}\right)$ be a real sequence and let $A=\left(a_{n j}\right)$ be a summability matrix. If the sequence $\left\{(A x)_{n}\right\}$ is convergent to a real number $L$, then we say that the sequence $x$ is $A$-summable to the real number $L$ where the series

$$
(A x)_{n}:=\sum_{j=0}^{\infty} a_{n j} x_{j}
$$

is convergent for any $n \in \mathbb{N}_{0}$ and $\mathbb{N}_{0}=\{0,1, \ldots\}$. A summability matrix $A$ is said to be regular if $\lim (A x)_{n}=L$ whenever $\lim x=L$ (see, $\left.[10]\right)$.

Let $A=\left(a_{n j}\right)$ be a non-negative regular summability matrix and let $E \subset \mathbb{N}_{0}$. Then the number

$$
\delta_{A}(E):=\lim \sum_{j \in E} a_{n j}
$$

is said to be the $A$-density of $E$ whenever the limit exists (see, 20$]$ ). Regularity of the summability matrix $A$ ensures that $0 \leq \delta_{A}(E) \leq 1$ whenever $\delta_{A}(E)$ exists. If we consider $A=C$, the Cesàro

2020 Mathematics Subject Classification: Primary 41A36, 40C15.

Keywords: Power series statistical convergence, Korovkin type theorem, Balázs type Bleimann, Butzer and Hahn operators. 


\section{DILEK SÖYLEMEZ}

matrix, then $\delta(E):=\delta_{C}(E)$ is called the (natural or asymptotic) density of $E$ (see, [15]) where $C=\left(c_{n j}\right)$ is the summability matrix defined by

$$
c_{n j}= \begin{cases}\frac{1}{n+1}, & \text { if } j \leq n, \\ 0, & \text { otherwise. }\end{cases}
$$

A real sequence $x=\left(x_{j}\right)$ is said to be $A$-statistically convergent (see, 16]) to a real number $L$ if for any $\varepsilon>0$,

$$
\delta_{A}\left(\left\{j \in \mathbb{N}_{0}:\left|x_{j}-L\right| \geq \varepsilon\right\}\right)=0 .
$$

In this case we write $s t_{A}-\lim x=L$. If we consider the Cesàro matrix, then $C$-statistical convergence is called statistical convergence [14]. In general, $A$-statistical convergence is regular and there exists some sequences which are $A$-statistically convergent but not ordinary convergent.

In what follows we recall the concept of power series method 10:

Let $\left(p_{j}\right)$ be a real sequence with $p_{0}>0$ and $p_{1}, p_{2}, \ldots \geq 0$, and such that the corresponding power series $p_{t}=\sum_{j=0}^{\infty} p_{j} t^{j}$ has radius of convergence $R$ with $0<R \leq \infty$. If for all $t \in(0, R)$,

$$
\lim _{t \rightarrow R^{-}} \frac{1}{p(t)} \sum_{j=0}^{\infty} x_{j} p_{j} t^{j}=L,
$$

then we say that $x=\left(x_{j}\right)$ is convergent in the sense of power series method $P_{p}$. Such a summability method is a general version of the Abel and Borel summability methods. Related to these methods, some approximation results can be found in [5, 9, 23, 24, 26, 27, 29.

The following theorem characterizes the regularity of a power series method.

Definition $1([10])$. A power series method $P_{p}$ is regular if and only if for any $j \in \mathbb{N}_{0}$

$$
\lim _{0<t \rightarrow R^{-}} \frac{p_{j} t^{j}}{p(t)}=0
$$

Ünver and Orhan 31] introduced the concept of $P_{p}$-statistical convergence. This type of convergence is based on the notion of the $P_{p}$-density of a subset of $\mathbb{N}_{0}$, we mention it as below:

Definition $2([31])$. Let $P_{p}$ be a regular power series method and let $E \subset \mathbb{N}_{0}$. If

$$
\delta_{P_{p}}(E):=\lim _{0<t \rightarrow R^{-}} \frac{1}{p(t)} \sum_{j \in E}^{\infty} p_{j} t^{j}
$$

exists, then $\delta_{P_{p}}(E)$ is called the $P_{p}$-density of $E$. Note that $\delta_{P_{p}}(E)$ satisfies the inequality $0 \leq \delta_{P_{p}}(E) \leq 1$, whenever it exists.

Definition $3([31])$. Let $x=\left(x_{j}\right)$ be a real sequence and let $P_{p}$ be a regular power series method. Then $x$ is said to be $P_{p}$-statistically convergent to $L$ if for any $\varepsilon>0$

$$
\lim _{0<t \rightarrow R^{-}} \frac{1}{p(t)} \sum_{\left|x_{j}-L\right| \geq \varepsilon}^{\infty} p_{j} t^{j}=0
$$

i.e., $\delta_{P_{p}}\left(\left\{j \in \mathbb{N}_{0}:\left|x_{j}-L\right| \geq \varepsilon\right\}\right)=0$ for any $\varepsilon>0$. In this case we write $s t_{P_{p}}-\lim x=L$.

In 1999, Gadjiev and Çakar [18 gave a Korovkin type theorem in ordinary sense, by using the test functions $\left(\frac{t}{1+t}\right)^{v}$ for $v=0,1,2$. In that research, the authors also investigated uniform approximation properties of the Bleimann, Butzer and Hahn (BBH) operators. Later, Erkuş 


\section{BALÁZS TYPE BLEIMANN, BUTZER AND HAHN OPERATORS}

and Duman [13 studied an extention of the Korovkin type theorem given by Gadjiev and Çakar considering $A$-statistical convergence in multivariate case and showed that the new theorem is quite useful. Aktuğlu and Özarslan 1 proved a Korovkin type theorem by using ideal convergence. They also introduced multivariate $\mathrm{B} B \mathrm{H}$ type operators and proved ideal convergence of sequence of these operators which doesn't converge in ordinary sense.

In the present paper, taking into account the $P_{p}$-statistical convergence, we obtain a Korovkin type approximation theorem in multivariable case. We also construct non-tensor multivariete Balázs type BBH operator $\left\{L_{n}\right\}$ which does not converge in ordinary sense. Furthermore, we prove the $P_{p}$-statistical convergence of these operators for the function $f$ belonging the space $H_{\omega}^{m}$ which is a subspace of continuous and bounded functions defined on $S$. Finally, we compute the rate of the $P_{p}$-statistical convergence of the operators $\left\{L_{n}\right\}$ by means of modulus of continuity function.

Now, we recall some notations of multivariate setting:

Let us consider the set $S \subset \mathbb{R}^{m},(m \in \mathbb{N})$, given by

$$
S=\left\{\mathbf{x}=\left(x_{1}, \ldots, x_{m}\right) \in \mathbb{R}^{m}: x_{i} \geq 0,1 \leq i \leq m\right\} .
$$

For $\mathbf{x}=\left(x_{1}, \ldots, x_{m}\right) \in S$ and $\mathbf{k}=\left(k_{1}, \ldots, k_{m}\right) \in \mathbb{N}^{m} \cup\{\mathbf{0}\}$, and $n \in \mathbb{N}$, we denote

$$
\begin{aligned}
|\mathbf{x}| & =\sum_{i=1}^{m} x_{i},|\mathbf{k}|:=k_{1}+k_{2}+\cdots+k_{m}, \quad \mathbf{k} !:=k_{1} ! k_{2} ! \cdots k_{m} !, \\
\mathbf{x}^{\mathbf{k}} & :=\left(x_{1}^{k_{1}} x_{2}^{k_{2}} \cdots x_{m}^{k_{m}}\right), \quad \mathbf{0}^{0}:=1, \quad \alpha \mathbf{x}:=\left(\alpha x_{1}, \ldots, \alpha x_{m}\right) \text { for } \alpha \in \mathbb{R}, \\
\left(\begin{array}{l}
n \\
\mathbf{k}
\end{array}\right) & :=\frac{n !}{\mathbf{k} !(n-|\mathbf{k}|) !} \quad \sum_{0 \leq|\mathbf{k}| \leq n}:=\sum_{k_{1}=0}^{n} \sum_{k_{2}=0}^{n-k_{1}} \cdots \sum_{k_{m}=0}^{n-k_{1}-\cdots-k_{m-1}} .
\end{aligned}
$$

The Euclidean norm of $\mathbf{x}=\left(x_{1}, \ldots, x_{m}\right)$ is given by $\|\mathbf{x}\|=\sqrt{\sum_{i=1}^{m} x_{i}^{2}}$.

Furthermore, we simply write $f(\mathbf{x})$ rather $f\left(x_{1}, \ldots, x_{m}\right)$ for $\mathbf{x}=\left(x_{1}, \ldots, x_{m}\right) \in S$, we also write

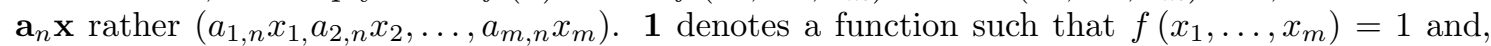
for any $\mathbf{x}=\left(x_{1}, \ldots, x_{m}\right), \mathbf{y}=\left(y_{1}, \ldots, y_{m}\right) \in S, \mathbf{x} \leq \mathbf{y}$ means that $x_{i} \leq y_{i}$ for each $i=1,2, \ldots, m$.

Let $C_{B}(S)$ denote the space of all real valued continuous and bounded functions defined on $S$, with norm

$$
\|f\|_{C_{B}}=\sup _{\mathbf{x} \in S}|f(\mathbf{x})| .
$$

Below, we give the definition of the modulus of continuity function.

Definition $4(\sqrt[4]{4})$. A non-negative function $\omega(\mathbf{u})$ defined in $S \subset \mathbb{R}^{m}$ is called a function of modulus of continuity, if it satisfies the following conditions for any $\boldsymbol{\delta}=\left(\delta_{1}, \ldots, \delta_{m}\right), \boldsymbol{\mu}=$ $\left(\mu_{1}, \ldots, \mu_{m}\right) \in S$ :

(1) $\omega(\boldsymbol{\delta})$ is continuous for all $\delta_{i}, i=1, \ldots, m$,

(2) $\omega(\mathbf{0})=0$, where $\mathbf{0}=(0,0, \ldots, 0)$,

(3) $\omega(\boldsymbol{\delta})=\omega\left(\delta_{1}, \ldots, \delta_{m}\right)$ is non-decreasing, i.e., $\omega(\boldsymbol{\delta}) \geq \omega(\boldsymbol{\mu})$ for $\boldsymbol{\delta} \geq \boldsymbol{\mu}$,

(4) $\omega(\boldsymbol{\delta})$ is sub-additive, i.e., $\omega(\boldsymbol{\delta}+\boldsymbol{\mu}) \leq \omega(\boldsymbol{\delta})+\omega(\boldsymbol{\mu})$.

Let $H_{\omega}^{m}(S)$ denote the space of all real valued functions defined on $S$ satisfying

$$
|f(\mathbf{x})-f(\mathbf{y})| \leq \omega\left(\left|\frac{x_{1}}{1+|\mathbf{x}|}-\frac{y_{1}}{1+|\mathbf{y}|}\right|, \ldots,\left|\frac{x_{m}}{1+|\mathbf{x}|}-\frac{y_{m}}{1+|\mathbf{y}|}\right|\right),
$$




\section{DILEK SÖYLEMEZ}

for all $\mathbf{x}=\left(x_{1}, \ldots, x_{m}\right), \mathbf{y}=\left(y_{1}, \ldots, y_{m}\right) \in S$. It is easy to see that $H_{\omega}^{m}(S) \subset C_{B}(S)$. Letting univariate modulus of continuity in (1.1), we obtain the space $H_{\omega}$ defined in 18.

In [25], Söylemez et al. used the class $H_{\omega}^{m}(S)$ to prove a Korovkin type theorem for uniform convergence. As an application of this theorem, the authors gave uniform approximation properties of non-tensor multivariate BBH operators. They also show that uniform convergence does not achieve when selecting a function from the space $C_{B}(S)$, instead of $H_{\omega}^{m}(S)$.

In 22, Özarslan et al. gave a Korovkin type theorem in multivariable case for Balázs-type BBH operators considering a class which was produced by univariate modulus of continuity function. In that research, the authors also obtained rate of the convergence for Bivariate case.

For $f \in C_{B}(S)$, we consider the following Balázs-type BBH operator which is not a tensor product setting.

$$
L_{n}(f ; \mathbf{x})=\frac{b_{n}}{\left(1+\left|\mathbf{a}_{n} \mathbf{x}\right|\right)^{n}} \sum_{0 \leq|\mathbf{k}| \leq n}\left(\begin{array}{l}
n \\
\mathbf{k}
\end{array}\right)\left(\mathbf{a}_{n} \mathbf{x}\right)^{\mathbf{k}} f\left(\frac{\mathbf{k}}{n+1-|\mathbf{k}|}\right),
$$

where $\mathbf{x}=\left(x_{1}, x_{2}, \ldots, x_{m}\right) \in S, b_{n} \geq 0, a_{i, n} \geq 0$ for all $i=1,2, \ldots, m$, and $n \in \mathbb{N}$.

Throughout the paper, we assume that $L_{0}(f)=0$ for any $f \in C_{B}(S)$ and we use the following test functions:

$$
\begin{aligned}
\hat{e}_{0}(\mathbf{x}) & =1 \\
\hat{e}_{i}(\mathbf{x}) & =\frac{x_{i}}{1+|\mathbf{x}|}, \\
\hat{e}_{m+1}(\mathbf{x}) & =\sum_{i=1}^{m}\left(\frac{x_{i}}{1+|\mathbf{x}|}\right)^{2} .
\end{aligned}
$$

The following example shows that there exists a sequence $\left(b_{n}\right)$ such that $P_{p}$-statistical convergence holds but ordinary convergence does not hold.

Example 1. We assume that $P_{p}$ is the regular power series method with $\left(p_{n}\right)$

$$
p_{n}:= \begin{cases}0, & n=2 k \\ 1, & n=2 k+1,\end{cases}
$$

and we consider the sequence $\left(b_{n}\right)$ such as

$$
b_{n}:= \begin{cases}n, & n=2 k \\ 1, & n=2 k+1\end{cases}
$$

it is not convergent, but $P_{p}$-statistical convergent.

If we take $m=1, b_{n}=1, n \in \mathbb{N}$, the operators 1.2 reduce to the Balázs type Bleimann Butzer and Hahn operators (see; 6, 7]).

\section{Main result}

In this section, using $P_{p}$-statistical convergence, we prove a Korovkin type theorem for the operators 1.2 . Now, we recall, the following Korovkin type theorem which was given by Gadjiev and Çakar 18 . 
TheоReм $2.1\left([18)\right.$. Let $\left(A_{n}\right)$ be a sequence of positive linear operators from $H_{\omega} \rightarrow C_{B}[0, \infty)$.

Then we have

$$
\lim _{n}\left\|A_{n}(f)-f\right\|_{C_{B}}=0
$$

if and only if

$$
\lim _{n}\left\|A_{n}\left(e_{i}\right)-e_{i}\right\|_{C_{B}}=0, i=0,1,2
$$

where $e_{i}(x)=\left(\frac{x}{1+x}\right)^{i}$.

Multivariate extension of Theorem 2.1 was proved in 25]. We remark that the sequence of the operators 1.2 does not satisfy the conditions of these two theorems. Therefore, it may be beneficial to use the following theorem.

TheOREM 2.2. Let $P_{p}$ regular power series method and let $\left\{R_{n}(f)\right\}_{n \in \mathbb{N}}$ be a sequence of linear positive operators from $H_{\omega}^{m}(S)$ to $C_{B}(S)$. If

$$
\begin{aligned}
& s t_{P_{p}}-\lim _{n}\left\|R_{n}\left(\hat{e}_{0}\right)-\hat{e}_{0}\right\|_{C_{B}}=0, \\
& s t_{P_{p}}-\lim _{n}\left\|R_{n}\left(\hat{e}_{i}\right)-\hat{e}_{i}\right\|_{C_{B}}=0 \quad \text { for all } i=1, \ldots, m+1
\end{aligned}
$$

are satisfied, then for $f \in H_{\omega}^{m}(S)$, we have

$$
s t_{P_{p}}-\lim _{n}\left\|R_{n}(f)-f\right\|_{C_{B}}=0 .
$$

Proof. It is clear that 2.2 implies 2.1). Suppose that $f \in H_{\omega}^{m}(S)$ and $\mathbf{x}=\left(x_{1}, \ldots, x_{m}\right)$, $\mathbf{t}=\left(t_{1}, \ldots, t_{m}\right)$ are any two elements of $S$. Then, from Definition 4 , for any given $\epsilon>0$, we can find a $\delta_{i}>0$, for $i=1,2, \ldots, m$ and taking $\delta=\min \left\{\delta_{1}, \delta_{2}, \ldots, \delta_{m}\right\}$, we may write

$$
|f(\mathbf{t})-f(\mathbf{x})|<\epsilon \text { whenever }\left|\frac{t_{i}}{1+|\mathbf{t}|}-\frac{x_{i}}{1+|\mathbf{x}|}\right|<\delta \quad(i=1,2, \ldots, m) .
$$

On the other hand, if $\left|\frac{t_{i_{0}}}{1+|\mathbf{t}|}-\frac{x_{i_{0}}}{1+|\mathbf{x}|}\right| \geq \delta$ for some $i_{0} \in\{1,2, \ldots, m\}$, then we have

$$
\left\|\frac{\mathbf{t}}{1+|\mathbf{t}|}-\frac{\mathbf{x}}{1+|\mathbf{x}|}\right\|=\sqrt{\sum_{i=1}^{m}\left(\frac{t_{i}}{1+|\mathbf{t}|}-\frac{x_{i}}{1+|\mathbf{x}|}\right)^{2}} \geq\left|\frac{t_{i_{0}}}{1+|\mathbf{t}|}-\frac{x_{i_{0}}}{1+|\mathbf{x}|}\right| \geq \delta .
$$

From the boundedness of $f$ on $S$, one has

$$
|f(\mathbf{t})-f(\mathbf{x})| \leq \frac{2\|f\|_{C_{B}}}{\delta^{2}}\left\|\frac{\mathbf{t}}{1+|\mathbf{t}|}-\frac{\mathbf{x}}{1+|\mathbf{x}|}\right\|^{2}
$$

whenever $\left|\frac{t_{i}}{1+|\mathbf{t}|}-\frac{x_{i}}{1+|\mathbf{x}|}\right| \geq \delta$ for some $i_{0} \in\{1,2, \ldots, m\}$. Therefore, for all $\mathbf{x}, \mathbf{t} \in S$ we can write

$$
\begin{aligned}
|f(\mathbf{t})-f(\mathbf{x})| & \leq \epsilon+\frac{2\|f\|_{C_{B}}}{\delta^{2}} \sum_{i=1}^{m}\left(\frac{t_{i}}{1+|\mathbf{t}|}-\frac{x_{i}}{1+|\mathbf{x}|}\right)^{2} \\
& =\epsilon+\frac{2\|f\|_{C_{B}}}{\delta^{2}}\left\|\frac{\mathbf{t}}{1+|\mathbf{t}|}-\frac{\mathbf{x}}{1+|\mathbf{x}|}\right\|^{2} .
\end{aligned}
$$

Application of the operators $\left(R_{n}\right)$ to 2.4 gives

$$
\left|R_{n}(f(\mathbf{t}) ; \mathbf{x})-f(\mathbf{x})\right| \leq\left(R_{n}(|f(\mathbf{t})-f(\mathbf{x})| ; \mathbf{x})\right)+\|f\|_{C_{B}}\left|\left(R_{n}(\mathbf{1} ; \mathbf{x})-1\right)\right| .
$$




\section{DILEK SÖYLEMEZ}

From the linearity and positivity of the operators $\left(R_{n}\right)$ and considering 2.1 , we have

$$
\begin{aligned}
\left|R_{n}(f(\mathbf{t}) ; \mathbf{x})-f(\mathbf{x})\right| \leq & \varepsilon+\left(\epsilon+\|f\|_{C_{B}}+\frac{2\|f\|_{C_{B}}}{\delta^{2}} m\right)\left|R_{n}\left(\hat{e}_{0}\right)-\hat{e}_{0}\right| \\
& +\frac{4\|f\|_{C_{B}}}{\delta^{2}} \sum_{i=1}^{m}\left|R_{n}\left(\hat{e}_{i}\right)-\hat{e}_{i}\right|+\frac{2\|f\|_{C_{B}}}{\delta^{2}}\left|R_{n}\left(\hat{e}_{m+1}\right)-\hat{e}_{m+1}\right|,
\end{aligned}
$$

which implies

$$
\left\|R_{n}(f)-f\right\|_{C_{B}} \leq \epsilon+K\left\{\sum_{i=0}^{m+1}\left\|R_{n}\left(\hat{e}_{i}\right)-\hat{e}_{i}\right\|_{C_{B}}\right\}
$$

where $K=\max \left\{\epsilon+\|f\|_{C_{B}}+\frac{2 m\|f\|_{C_{B}}}{\delta^{2}}, \frac{4\|f\|_{C_{B}}}{\delta^{2}}\right\}$.

For a given $s>0$ we select $\epsilon>0$ such that $\epsilon<s$. Let us define the following sets

$$
\begin{aligned}
U & :=\left\{n \in \mathbb{N}:\left\|R_{n}(f)-f\right\|_{C_{B}} \geq s\right\}, \\
U_{i} & :=\left\{n \in \mathbb{N}:\left\|R_{n}\left(\hat{e}_{i}\right)-\hat{e}_{i}\right\|_{C_{B}} \geq \frac{s-\epsilon}{K(m+2)}\right\}, \quad i=0,1,2, \ldots, m+1 .
\end{aligned}
$$

Then, by (2.1), we have $U \subset\left(\bigcup_{i=0}^{m+1} U_{i}\right)$. Hence, for all $n \in \mathbb{N}$,

$$
0 \leq \delta_{P_{p}}\left\{n \in \mathbb{N}:\left\|R_{n}(f)-f\right\|_{C_{B}} \geq s\right\} \leq \sum_{i=0}^{m+1} \delta_{P_{p}}\left(U_{i}\right)=0,
$$

which completes the proof.

\section{Power series statistical convergence of the operators $\left(L_{n}\right)$}

In this section, we investigate the power series statistical convergence properties of the operators (1.2) and compute the rate of the $P_{p}$-statistical convergence by means of modulus of continuity [4] Before studying the promised approximation properties of these operators, we give the following lemma which can be proved as in 25 .

\section{LeMma 3.1.}

$$
\begin{aligned}
L_{n}(\mathbf{1} ; \mathbf{x}) & =b_{n}, \\
L_{n}\left(\left(\frac{t_{i}}{1+|\mathbf{t}|}\right) ; \mathbf{x}\right) & =b_{n} \frac{n}{n+1}\left(\frac{a_{i, n} x_{i}}{1+\left|\mathbf{a}_{n} \mathbf{x}\right|}\right) \quad \text { for } i=1, \ldots, m, \\
L_{n}\left(\sum_{i=1}^{m}\left(\frac{t_{i}}{1+|\mathbf{t}|}\right)^{2} ; \mathbf{x}\right) & =b_{n} \sum_{i=1}^{m}\left\{\frac{n(n-1)}{(n+1)^{2}}\left(\frac{a_{i, n} x_{i}}{1+\left|\mathbf{a}_{n} \mathbf{x}\right|}\right)^{2}+\frac{n}{(n+1)^{2}} \frac{a_{i, n} x_{i}}{1+\left|\mathbf{a}_{n} \mathbf{x}\right|}\right\} .
\end{aligned}
$$

In the following theorem, we investigate the power series statistical convergence properties of the sequence $\left(L_{n}\right)$ for any $f \in H_{\omega}^{m}(S)$ on $S$.

THEOREM 3.1. Let $\left(L_{n}\right)$ be the sequence of the operators defined by $(1.2)$ and suppose that $s t_{P_{p}}-\lim _{n} b_{n}=1, s t_{P_{p}}-\lim _{n} a_{i, n}=1$, for all $i=1,2, \ldots, m$. Then for any $f \in H_{\omega}^{m}(S)$ we have

$$
s t_{P_{p}}-\lim _{n}\left\|L_{n}(f)-f\right\|_{C_{B}}=0 .
$$


Pr o of. From Theorem 2.2, it suffices to show that 2.1p holds for $\left(L_{n}\right)$. Indeed, from Lemma 3.1 and the hypothesis, we have

$$
s t_{P_{p}}-\lim _{n}\left\|L_{n}\left(\hat{e}_{0}\right)-\hat{e}_{0}\right\|_{C_{B}}=0 .
$$

Also, for all $i=1,2, \ldots, m$, we can write

$$
\begin{aligned}
& \left|L_{n}\left(\left(\frac{t_{i}}{1+|\mathbf{t}|}\right) ; \mathbf{x}\right)-\frac{x_{i}}{1+|\mathbf{x}|}\right| \\
& =\left|b_{n} \frac{n}{n+1}\left(\frac{a_{i, n} x_{i}}{1+\left|\mathbf{a}_{n} \mathbf{x}\right|}\right)-\frac{x_{i}}{1+|\mathbf{x}|}\right| \\
& =\left|b_{n} \frac{n}{n+1}\left(a_{i, n} x_{i}\right)\left(\frac{1}{1+\left|\mathbf{a}_{n} \mathbf{x}\right|}-\frac{1}{1+|\mathbf{x}|}\right)+\left(\frac{x_{i}}{1+|\mathbf{x}|}\right)\left(b_{n} \frac{n}{n+1}\left(a_{i, n}\right)-1\right)\right| \\
& \leq b_{n}\left(a_{i, n} x_{i}\right) \frac{|| \mathbf{x}|-| \mathbf{a}_{n} \mathbf{x} \mid}{\left(1+\left|\mathbf{a}_{n} \mathbf{x}\right|\right)(1+|\mathbf{x}|)}+\left(\frac{x_{i}}{1+|\mathbf{x}|}\right)\left|b_{n} \frac{n}{n+1}\left(a_{i, n}\right)-1\right| \\
& \leq b_{n}\left(a_{i, n} x_{i}\right) \frac{|\mathbf{x}|\left(\sum_{i=1}^{m}\left|1-a_{i, n}\right|\right)}{\left(1+\left|\mathbf{a}_{n} \mathbf{x}\right|\right)(1+|\mathbf{x}|)}+\left(\frac{x_{i}}{1+|\mathbf{x}|}\right)\left|b_{n} \frac{n}{n+1}\left(a_{i, n}\right)-1\right| \\
& =b_{n}\left(\sum_{i=1}^{m}\left|1-a_{i, n}\right|\right)\left(\frac{a_{i, n} x_{i}}{1+\left|\mathbf{a}_{n} \mathbf{x}\right|}\right)\left(\frac{|\mathbf{x}|}{1+|\mathbf{x}|}\right)+\left(\frac{x_{i}}{1+|\mathbf{x}|}\right)\left|b_{n}\left(a_{i, n}\right) \frac{n}{n+1}-1\right| \\
& \leq b_{n}\left(\sum_{i=1}^{m}\left|1-a_{i, n}\right|\right)+\left|\left(b_{n}\left(a_{i, n}\right) \frac{n}{n+1}-1\right)\right| .
\end{aligned}
$$

Thus, we reach to

$$
\left\|L_{n}\left(\hat{e}_{i}\right)-\hat{e}_{i}\right\|_{C_{B}} \leq b_{n}\left(\sum_{i=1}^{m}\left|1-a_{i, n}\right|\right)+\left|b_{n} \frac{n}{n+1}\left(a_{i, n}\right)-1\right|,
$$

for all $i=1,2, \ldots, m$. Now, let us define the following sets for any $\epsilon>0$,

$$
\begin{aligned}
N_{i} & :=\left\{n \in \mathbb{N}:\left\|L_{n}\left(\hat{e}_{i}\right)-\hat{e}_{i}\right\|_{C_{B}} \geq \epsilon\right\}, \\
N_{i}^{1} & :=\left\{n \in \mathbb{N}:\left|b_{n} \sum_{i=1}^{m}\right| 1-a_{i, n}|| \geq \frac{\epsilon}{2}\right\}, \\
N_{i}^{2} & :=\left\{n \in \mathbb{N}:\left|\left(b_{n} \frac{n}{n+1}\left(a_{i, n}\right)-1\right)\right| \geq \frac{\epsilon}{2}\right\},
\end{aligned}
$$

for $i=1, \ldots, m$, it is obvious that $N_{i} \subset N_{i}^{1} \cup N_{i}^{2}$. Therefore, we can write

$$
\begin{aligned}
0 \leq & \delta_{P_{p}}\left\{n \in \mathbb{N}:\left\|L_{n}\left(\hat{e}_{i}\right)-\hat{e}_{i}\right\|_{C_{B}} \geq \epsilon\right\} \\
\leq & \delta_{P_{p}}\left\{n \in \mathbb{N}:\left|b_{n}\left(\sum_{i=1}^{m}\left|1-a_{i, n}\right|\right)\right| \geq \frac{\epsilon}{2}\right\} \\
& +\delta_{P_{p}}\left\{n \in \mathbb{N}:\left|b_{n} \frac{n}{n+1}\left(a_{i, n}\right)-1\right| \geq \frac{\epsilon}{2}\right\},
\end{aligned}
$$

for $i=1, \ldots, m$. By the assumptions and $s t_{P_{p}}-\lim _{n} \frac{n}{n+1}=1$, we have

$$
0 \leq \delta_{P_{p}}\left\{n \in \mathbb{N}:\left\|L_{n}\left(\hat{e}_{i}\right)-\hat{e}_{i}\right\|_{C_{B}} \geq \epsilon\right\}=0 .
$$




\section{DILEK SÖYLEMEZ}

On the other hand, from 3.3 , we can write

$$
\begin{aligned}
& \left|L_{n}\left(\sum_{i=1}^{m}\left(\frac{t_{i}}{1+|\mathbf{t}|}\right)^{2} ; \mathbf{x}\right)-\sum_{i=1}^{m}\left(\frac{x_{i}}{1+|\mathbf{x}|}\right)^{2}\right| \\
& =\left|b_{n} \sum_{i=1}^{m}\left\{\frac{n(n-1)}{(n+1)^{2}}\left(\frac{a_{i, n} x_{i}}{1+\left|\mathbf{a}_{n} \mathbf{x}\right|}\right)^{2}+\frac{n}{(n+1)^{2}} \frac{a_{i, n} x_{i}}{1+\left|\mathbf{a}_{n} \mathbf{x}\right|}\right\}-\sum_{i=1}^{m}\left(\frac{x_{i}}{1+|\mathbf{x}|}\right)^{2}\right| \\
& \leq\left|b_{n} \sum_{i=1}^{m} \frac{n(n-1)}{(n+1)^{2}}\left(\frac{a_{i, n} x_{i}}{1+\left|\mathbf{a}_{n} \mathbf{x}\right|}\right)^{2}-\sum_{i=1}^{m}\left(\frac{x_{i}}{1+|\mathbf{x}|}\right)^{2}\right|+\left|b_{n} \sum_{i=1}^{m} \frac{n}{(n+1)^{2}} \frac{a_{i, n} x_{i}}{1+\left|\mathbf{a}_{n} \mathbf{x}\right|}\right| \\
& :=A_{1}+A_{2} .
\end{aligned}
$$

It follows that

$$
\begin{aligned}
A_{1} \leq & \left|b_{n} \frac{n(n-1)}{(n+1)^{2}} \sum_{i=1}^{m}\left(a_{i, n} x_{i}\right)^{2}\left(\frac{1}{\left(1+\left|\mathbf{a}_{n} \mathbf{x}\right|\right)^{2}}-\frac{1}{(1+|\mathbf{x}|)^{2}}\right)\right| \\
& +\left|\sum_{i=1}^{m}\left(\frac{x_{i}}{1+|\mathbf{x}|}\right)^{2}\left(b_{n} \frac{n(n-1)}{(n+1)^{2}}\left(a_{i, n}\right)^{2}-1\right)\right| \\
\leq & b_{n} \sum_{i=1}^{m}\left(a_{i, n} x_{i}\right)^{2}\left|\frac{1}{\left(1+\left|\mathbf{a}_{n} \mathbf{x}\right|\right)^{2}}-\frac{1}{(1+|\mathbf{x}|)^{2}}\right| \\
& +\left|\sum_{i=1}^{m}\left(\frac{x_{i}}{1+|\mathbf{x}|}\right)^{2}\left(b_{n} \frac{n(n-1)}{(n+1)^{2}}\left(a_{i, n}\right)^{2}-1\right)\right| \\
\leq & b_{n} \sum_{i=1}^{m}\left(a_{i, n} x_{i}\right)^{2} \frac{\left.|| \mathbf{x}\right|^{2}-\left|\mathbf{a}_{n} \mathbf{x}\right|^{2} \mid}{\left(1+\left|\mathbf{a}_{n} \mathbf{x}\right|\right)^{2}(1+|\mathbf{x}|)^{2}} \\
& +b_{n} \frac{n(n-1)}{(n+1)^{2}} \sum_{i=1}^{m}\left(a_{i, n} x_{i}\right)^{2} \frac{\left|2\left(|\mathbf{x}|-\left|\mathbf{a}_{n} \mathbf{x}\right|\right)\right|}{\left(1+\left|\mathbf{a}_{n} \mathbf{x}\right|\right)^{2}(1+|\mathbf{x}|)^{2}} \\
& +\sum_{i=1}^{m}\left(\frac{x_{i}}{1+|\mathbf{x}|}\right)^{2}\left|b_{n} \frac{n(n-1)}{(n+1)^{2}}\left(a_{i, n}\right)^{2}-1\right|
\end{aligned}
$$

Taking into account the inequalities

$$
|| \mathbf{x}|-| \mathbf{a}_{n} \mathbf{x}|| \leq|\mathbf{x}| \sum_{i=1}^{m}\left|1-a_{i, n}\right| \quad \text { and }\left.\quad|| \mathbf{x}\right|^{2}-\left.\left|\mathbf{a}_{n} \mathbf{x}\right|^{2}|\leq| \mathbf{x}\right|^{2}\left(\sum_{i=1}^{m}\left|1-a_{i, n}\right|\right)\left(m+\left|\mathbf{a}_{n}\right|\right)
$$

we get

$$
\begin{aligned}
A_{1} \leq & b_{n} \sum_{i=1}^{m} \frac{\left(a_{i, n} x_{i}\right)^{2}}{\left(1+\left|\mathbf{a}_{n} \mathbf{x}\right|\right)^{2}} \frac{|\mathbf{x}|^{2}}{(1+|\mathbf{x}|)^{2}}\left(\sum_{i=1}^{m}\left|1-a_{i, n}\right|\right)\left(m+\left|\mathbf{a}_{n}\right|\right) \\
& +2 b_{n} \sum_{i=1}^{m} \frac{\left(a_{i, n} x_{i}\right)^{2}}{\left(1+\left|\mathbf{a}_{n} \mathbf{x}\right|\right)^{2}} \frac{|\mathbf{x}|}{(1+|\mathbf{x}|)^{2}}\left(\sum_{i=1}^{m}\left|1-a_{i, n}\right|\right) \\
& +\left|\sum_{i=1}^{m}\left(\frac{x_{i}}{1+|\mathbf{x}|}\right)^{2}\left(b_{n} \frac{n(n-1)}{(n+1)^{2}}\left(a_{i, n}\right)^{2}-1\right)\right|
\end{aligned}
$$


Also, we have

$$
A_{2} \leq\left|b_{n} \sum_{i=1}^{m} \frac{n}{(n+1)^{2}} \frac{a_{i, n} x_{i}}{1+\left|\mathbf{a}_{n} \mathbf{x}\right|}\right| \leq \sum_{i=1}^{m} b_{n} \frac{n}{(n+1)^{2}} .
$$

Therefore, we reach to

$$
\begin{aligned}
& \left\|L_{n}\left(\hat{e}_{m+1}\right)-\hat{e}_{m+1}\right\|_{C_{B}} \\
& =\left|L_{n}\left(\sum_{i=1}^{m}\left(\frac{t_{i}}{1+|\mathbf{t}|}\right)^{2} ; \mathbf{x}\right)-\sum_{i=1}^{m}\left(\frac{x_{i}}{1+|\mathbf{x}|}\right)^{2}\right| \\
& \leq \sum_{i=1}^{m}\left\{m b_{n}\left|1-a_{i, n}\right|\left(m+\left|\mathbf{a}_{n}\right|\right)+2 m b_{n}\left|1-a_{i, n}\right|\right. \\
& \left.\quad+m\left|b_{n} \frac{n(n-1)}{(n+1)^{2}}\left(a_{i, n}\right)^{2}-1\right|+b_{n} \frac{n}{(n+1)^{2}}\right\} .
\end{aligned}
$$

Now, we define the following sets for any $\epsilon>0$ that

$$
\begin{aligned}
K & :=\left\{n \in \mathbb{N}: \|\left.\left(L_{n}\left(\hat{e}_{m+1}\right)-\hat{e}_{m+1}\right)\right|_{C_{B}} \geq \epsilon\right\}, \\
K_{i}^{1} & :=\left\{n \in \mathbb{N}:\left|m b_{n}\right| 1-a_{i, n}\left|\left(m+\left|\mathbf{a}_{n}\right|\right)\right| \geq \frac{\epsilon}{4 m}\right\}, \\
K_{i}^{2} & :=\left\{n \in \mathbb{N}:\left|2 m b_{n}\right| 1-a_{i, n}|| \geq \frac{\epsilon}{4 m}\right\}, \\
K_{i}^{3} & :=\left\{n \in \mathbb{N}:\left|b_{n} \frac{n(n-1)}{(n+1)^{2}}\left(a_{i, n}\right)^{2}-1\right| \geq \frac{\epsilon}{4 m}\right\}, \\
K_{i}^{4} & :=\left\{n \in \mathbb{N}:\left|b_{n} \frac{n}{(n+1)^{2}}\right| \geq \frac{\epsilon}{4 m}\right\},
\end{aligned}
$$

we easily see that $K \subset \bigcup_{i=1}^{m}\left(K_{i}^{1} \cup K_{i}^{2} \cup K_{i}^{3} \cup K_{i}^{4}\right)$. Finally we have

$$
\begin{aligned}
& \delta_{P_{p}}\left(\left\{n:\left\|L_{n}\left(\hat{e}_{m+1}\right)-\hat{e}_{m+1}\right\|_{C_{B}} \geq \epsilon\right\}\right) \\
& \leq \sum_{i=1}^{m}\left[\delta_{P_{p}}\left(\left\{n:\left|m b_{n}\right| 1-a_{i, n}\left|\left(m+\left|\mathbf{a}_{n}\right|\right)\right| \geq \frac{\epsilon}{4 m}\right\}\right)\right. \\
& \quad+\delta_{P_{p}}\left(\left\{n:\left|2 m b_{n}\right| 1-a_{i, n} \| \geq \frac{\epsilon}{4 m}\right\}\right) \\
& \quad+\delta_{P_{p}}\left(\left\{n:\left|b_{n} \frac{n(n-1)}{(n+1)^{2}}\left(a_{i, n}\right)^{2}-1\right| \geq \frac{\epsilon}{4 m}\right\}\right) \\
& \left.\quad+\delta_{P_{p}}\left(\left\{n:\left|b_{n} \frac{n}{(n+1)^{2}}\right| \geq \frac{\epsilon}{4 m}\right\}\right)\right] .
\end{aligned}
$$

Since $s t_{P_{p}}-\lim _{n} b_{n}=1, s t_{P_{p}}-\lim _{n} a_{i, n}=1$ for all $i=1,2, \ldots, m$ and $s t_{P_{p}}-\lim _{n} \frac{n(n-1)}{(n+1)^{2}}=1$, we get

$$
0 \leq \delta_{P_{p}}\left(\left\{n:\left\|L_{n}\left(\hat{e}_{m+1}\right)-\hat{e}_{m+1}\right\|_{C_{B}} \geq \epsilon\right\}\right)=0 .
$$

Thus, the proof is completed.

Now, we calculate the rate of $P_{p}$-statistical convergence via modulus of continuity function. The modulus of continuity function $\omega$ given in Definition 4 satisfies the inequality

$$
\omega(\lambda \boldsymbol{\delta}) \leq \omega(1+\lfloor\lambda\rfloor \boldsymbol{\delta}) \leq(1+\lambda) \omega(\boldsymbol{\delta})
$$




\section{DILEK SÖYLEMEZ}

where $\lfloor\lambda\rfloor$ is the greatest integer of $\lambda$.

Here, we are ready to give the following theorem:

THEOREм 3.2. Let $\left(L_{n}\right)$ be a sequence of positive linear operators defined by 1.2 . If

(i) $s t_{P_{p}}-\lim _{n}\left\|L_{n}\left(\hat{e}_{0}\right)-1\right\|_{C_{B}}=0$,

(ii) $s t_{P_{p}}-\lim _{n} \omega\left(\boldsymbol{\delta}_{n}\right)=0$,

then, for any $f \in H_{\omega}^{m}(S)$ we have

$$
s t_{P_{p}}-\lim _{n}\left\|L_{n}(f)-f\right\|_{C_{B}}=0,
$$

where

$$
\delta_{n}:=\left(\sup _{\mathbf{x} \in \mathbf{S}} L_{n}\left(\left\|\frac{\mathbf{t}}{1+|\mathbf{t}|}-\frac{\mathbf{x}}{1+|\mathbf{x}|}\right\|^{2} ; \mathbf{x}\right)\right)^{\frac{1}{2}} .
$$

Proof. Selecting $\lambda=\frac{\left\|\frac{\mathbf{t}}{1+|\mathbf{t}|}-\frac{\mathbf{x}}{1+|\mathbf{x}|}\right\|}{\delta}, \delta>0$ in 3.4 , for any $f \in H_{\omega}^{m}(S)$, we obtain

$$
\begin{aligned}
& \left|L_{n}(f ; \mathbf{x})-f(\mathbf{x})\right| \\
& \leq L_{n}(|f(\mathbf{t})-f(\mathbf{x})| ; \mathbf{x})+\|f\|_{C_{B}}\left|L_{n}\left(\hat{e}_{0}(\mathbf{t})\right)-1\right| \\
& \leq L_{n}\left(\left(1+\left\lfloor\frac{\left\|\frac{\mathbf{t}}{1+|\mathbf{t}|}-\frac{\mathbf{x}}{1+|\mathbf{x}|}\right\|}{\delta}\right\rfloor\right) \omega(\boldsymbol{\delta}) ; \mathbf{x}\right)+M\left|L_{n}\left(\hat{e}_{0}(\mathbf{t})\right)-1\right| \\
& \leq \omega(\boldsymbol{\delta}) L_{n}\left(\left(\hat{e}_{0}(\mathbf{t})\right) ; \mathbf{x}\right)+\frac{1}{\delta^{2}} \omega(\boldsymbol{\delta}) L_{n}\left(\left\|\frac{\mathbf{t}}{1+|\mathbf{t}|}-\frac{\mathbf{x}}{1+|\mathbf{x}|}\right\|^{2} ; \mathbf{x}\right)+M \mid L_{n}\left(\hat{e}_{0}(\mathbf{t})-1 \mid,\right.
\end{aligned}
$$

where $\omega(\boldsymbol{\delta})$ such that $\delta_{i}=\delta$, for $i=1,2, \ldots, m$ ( that is; $\omega(\boldsymbol{\delta})=\omega(\delta, \delta, \ldots, \delta)$ ). From (3.1), we obtain

$$
\begin{aligned}
\left|L_{n}(f ; \mathbf{x})-f(\mathbf{x})\right| \leq & \omega(\boldsymbol{\delta})+\omega(\boldsymbol{\delta})\left|b_{n}-1\right| \\
& +\frac{1}{\delta^{2}} \omega(\boldsymbol{\delta}) L_{n}\left(\left\|\frac{\mathbf{t}}{1+|\mathbf{t}|}-\frac{\mathbf{x}}{1+|\mathbf{x}|}\right\|^{2} ; \mathbf{x}\right)+M\left|b_{n}-1\right| \\
\leq & 2 \omega(\boldsymbol{\delta})+\omega(\boldsymbol{\delta})\left|b_{n}-1\right|+M\left|b_{n}-1\right| .
\end{aligned}
$$

Now, if we take $\delta_{n}=\left\{\sup _{\mathbf{x} \in \mathbf{S}} L_{n}\left(\left\|\frac{\mathbf{t}}{1+|\mathbf{t}|}-\frac{\mathbf{x}}{1+|\mathbf{x}|}\right\|^{2} ; \mathbf{x}\right)\right\}^{\frac{1}{2}}$, then we get

$$
0 \leq\left\|L_{n}(f)-f\right\| \leq 2 \omega\left(\boldsymbol{\delta}_{n}\right)+\omega\left(\boldsymbol{\delta}_{n}\right)\left|b_{n}-1\right|+M\left|b_{n}-1\right| .
$$

From (i) and (ii), we have

$$
s t_{P_{p}}-\lim _{n}\left\|L_{n}(f)-f\right\|_{C_{B}}=0
$$

which completes the proof.

\section{Concluding remarks}

It should be remarked that Theorem 2.1 and multivariate extension of this theorem given in 25 do not work for the operators 1.2 , but Theorem 2.2 works. So we can demonstrate that Theorem 2.2 is more powerful than Theorem 2.1 .

Acknowledgement. The author is grateful to the anonymous reviewers for valuable suggestions which greatly improved this paper. 


\title{
BALÁZS TYPE BLEIMANN, BUTZER AND HAHN OPERATORS
}

\author{
REFERENCES
}

[1] AKTUĞLU, H.-ÖZARSLAN, M. A.: Korovkin-type approximaton theorem for Bleimann, Butzer and Hahn Operators via I-onvergence, Math. Slovaca 60(6) (2010), 865-876.

[2] ALTOMARE, F.-CAMPITI, M.: Korovkin-type Approximaton Theory and its Applications, Walter de Gruyter, Berlin-New York, 1994.

[3] ANASTASSIOU, G. A.-DUMAN, O.: On Relaxing the positivity condition of linear operators in statistical Korovkin-type approximations, J. Comput. Appl. Math. 11(1) (2009), 7-19.

[4] ANDERSON, R.-BABENKO, Y.-LESKEVYCH, T.: Simultaneous approximation of a multivariate function and its derivatives by multilinear splines, J. Approx. Theory 183 (2014), 82-97.

[5] ATLIHAN, Ö. G.-ÜNNVER, M.: Abel transforms of convolution operators, Georgian Math. J. 22(3) (2015), 323-329.

[6] BALÁZS, K.: Approximation by Bernstein type rational functions, Acta Math. Acad. Sci. Hungar. 26(1-2) (1975), 123-134.

[7] BALÁZS, C.-SZABADOS, J.: Approximation by Bernstein type rational functions, Acta Math. Acad. Sci. Hungar. 40 (1982), 331-337.

[8] BALSER, W.: Formal Power Series and Linear Systems of Meromorphic Ordinary Differential Equations, Springer-Verlag, New York, 2000.

[9] BRAHA N. L.: Some properties of Baskakov-Schurer-Szász operators via power summability methods, Quaest. Math. 42(10) (2019), 1411-1426.

[10] BOOS, J.: Classical and Modern Methods in Summability, Oxford Univ. Press, UK 2000.

[11] DEMIRCI, K.-YILDIZ-S.-DIRIK, F.: Approximation via Power Series Method in Two-Dimensional Weighted Spaces, Bull. Malays. Math. Sci. Soc. 43 (2020), 3871-3883.

[12] DUMAN, O.: A Korovkin type approximation theorems via I-convergence, Czechoslovak Math. J. 57(132) (2007), 367-375.

[13] ERKUŞ, E.-DUMAN, O.: A-statistical extension of the Korovkin type approximation theorem, Proc. Indian Acad. Sci. Math. Sci. 115 (2005), 499-508.

[14] FAST, H.: Sur la convergence statistique, Colloq. Math. 2 (1951), 241-244.

[15] FREEDMAN, A. R.-SEMBER J. J.: Densities and summability, Pacific J. Math. 95(2) (1981), 293-305.

[16] FRIDY, J. A.-MILLER, H. I.: A matrix characterization of statistical convergence, Analysis 11(1) (1991), 59-66.

[17] GADJIEV, A. D.-ORHAN, C.: Some approximation theorems via statistical convergence, Rocky Mountain J. Math. 32 (2002), 129-138.

[18] GADJIEV, A. D.-ÇAKAR, Ö.: On uniform approximation by Bleimann, Butzer and Hahn Operators on all positive semiaxis, Trans. Natl. Acad. Sci. Azerb. Ser. Phys.-Tech. Math. Sci. 19(5) (1999), 21-26.

[19] GIULIANO ANTONINI, R.-ÜNVER, M.-VOLODIN, A. : On the concept of A-statistical uniform integrability and the law of large numbers, Lobachevskii J. Math. 40(12) (2019), 2034-2042.

[20] KOLK, E.: Matrix summability of statistically convergent sequences, Analysis 13(1-2) (1993), 77-83.

[21] ÖZARSLAN, M. A.-DUMAN, O.-SRIVASTAVA, H. M.: Statistical approximation results for Kantorovichtype operators involving some special polynomials, Math. Comput. Model. 48(3-4) (2008), 388-401.

[22] ÖZARSLAN, M. A.-AKTUĞLU, H.: Korovkin type theorem for non-tensor Balázs type Bleimann, Butzer and Hahn operators, Math. Meth. Appl. Sci. 38 (2015), 1937-1944.

[23] SÖYLEMEZ, D.-Ü̈NVER, M.: Korovkin type theorems for Cheney-Sharma Operators via summability methods, Res. Math. 73(3) (2017), 1601-1612.

[24] SÖYLEMEZ, D.-ÜNVER, M.: Korovkin type approximation of Abel transforms of q-Meyer König and Zeller operators, Int. J. Nonlinear Anal. Appl. 11(2) (2020), 339-350.

[25] SÖYLEMEZ, D.-AYDIN-ARI, D.-BAŞCANBAZ-TUNCA, G.: On multivariate Bleimann, Butzer and Hahn operators, Mediterr. J. Math. 17 (2020), 191.

[26] TAŞ, E.-YURDAKADIM, T.: Approximation to derivatives of functions by linear operators acting on weighted spaces by power series method. Computational Analysis, Springer Proceedings in Mathematics and Statistics 155, 2016, pp. 363-372.

[27] TAŞ, E.-YURDAKADIM, T.-ATLIHAN, Ö. G.: Korovkin type approximation theorems in weighted spaces via power series method, Oper. Matr. 12(2) (2018), 529-535.

[28] ÜNVER, M.: Abel transforms of positive linear operators. In: ICNAAM 2013. AIP Conference Proceedings 1558, 2013, pp. 1148-1151.

[29] ÜNVER, M.: Abel transforms of positive linear operators on weighted spaces, Bull. Belg. Math. Soc. Simon Stevin 21(5) (2014), 813-822. 


\section{DILEK SÖYLEMEZ}

[30] ÜNVER, M.-ULUÇAY, H.: Compactly uniform Bochner integrability of random elements, Positivity 21(4) (2017), 1261-1272.

[31] ÜNVER, M.-ORHAN, C.: Statistical Convergence with respect to power series methods and applications to approximation theory, J. Numer. Func. Anal. Optim. 40(5) (2019), 535-547.

Received 12. 6. 2020

Department of Mathematics

Accepted 28. 3. 2021

Faculty of Science

Selcuk University

Selcuklu 42003 Konya

TURKEY

E-mail: dsozden@gmail.com 